\title{
OSTEOCONDRITE DISSECANTE DA CABEÇA DO ÚMERO EM CÃES. ESTUDO RETROSPECTIVO DE 36 CASOS (1991-1996)
}

\author{
OSTEOCHONDRITIS DISSECANS OF THE HUMERAL HEAD IN DOGS. \\ RETROSPECTIVE STUDY OF 36 CASES (1991-1996)
}

\author{
André Luis Selmi ${ }^{1}$ João Guilherme Padilha Filho ${ }^{2}$ \\ Patrícia Mendes Pereira ${ }^{3}$ Cristina Mendes Batista ${ }^{1}$
}

RESUMO

A osteocondrite dissecante da cabeça do úmero (OCD) é uma condição patológica da cartilagem articular. decorrente de distúrbio da ossificação endocondral. Foram analisados 36 casos de OCD em cães com idades compreendidas entre 5 e 24 meses, observando-se maior representação entre machos comparados com fêmeas (3,5:I). A maioria destes animais (80,6\%) tinha recebido suplementação alimentar. Oito cães foram tratados conservativamente através de repouso e restrição alimentar, enquanto os demais foram submetidos a intervenção cirúrgica por meio de artrotomia e remoção do retalho de superficie articular da cabeça do úmero. Concluiu-se que a predisposição de algumas raças, associada ao desequilibrio nutricional durante os primeiros meses de vida, são as causas determinantes da OCD. e que a cirurgia é a melhor terapia a ser empregada.

Palavras-chave: osteocondrose, osteocondrite dissecante, articulação escápulo-umeral, cães.

\section{SUMMARY}

Osteocondritis dissecans of the humeral head (OCD) is a pathological condition of the articular cartilage, resulting from disturbance of the endochondral ossification. Thirty-six cases of $O C D$ were analyzed in dogs with ages between 5 and 24 months, with larger representation among males when compared to females (3.5:1). Most of these (80.6\%) had received nutritional suplementation. Eight dogs were treated conservatively through rest and

\begin{abstract}
dietary restriction, while the others were submitted to surgical intervention by means of arthrotomy and removal of the articular flap of the humeral head. It was concluded that the predisposition of some races, associated to the unbalanced nutritional diet during the first months of life, are the main causes of $O C D$, and that the surgery is the best therapy to be used.
\end{abstract}

Key words: ostechondrosis. osteochondritis dissecans, shoulder joint, dogs.

\section{INTRODUÇÃO}

A osteocondrite dissecante da cabeça do úmero é uma das manifestações clínicas da osteocondrose, representando condição patológica da cartilagem articular resultante de distúrbio da ossificação endocondral, que acomete cães de rápido crescimento e especialmente de grande porte, apesar de também ser observada em animais de porte médio. Sua maior incidência é observada em cães entre 4 e 8 meses de idade (FOX \& WALKER, 1993b; OLSSON, 1993; HOULTON \& COLLINSON, 1994).

RUDD et al. (1990), estudando 44 casos, observaram maior incidência em animais com peso corpóreo entre 25 e $50 \mathrm{~kg}$. Machos, que tendem a

\footnotetext{
${ }^{1}$ Pós-graduando em Cirurgia Veterinária, Departamento de Clínica e Cirurgia Veterinária (DCCV), Faculdade de Ciências Agrárias e Veterinária de Jaboticabal (FCAVJ), UNESP, Rodovia Carlos Tonanni, km 5, 14870-000, Jaboticabal/SP. Autor para correspondência.

${ }^{2}$ Professor Assistente, Dr, DCCV, FCAVJ, UNESP.

${ }^{3}$ Pós-graduada em Patologia Veterinária, DCCV, FCAVJ, UNESP. 
crescer mais rapidamente que fêmeas, e animais submetidos a dietas que promovem crescimento rápido são mais freqüentemente afetados, numa proporção que pode variar de 2 a 5 : 1 (FOX \& WALKER, 1993b).

As alterações ósseas decorrentes da ingestão de dietas ricas em proteínas, energia, cálcio e fósforo podem estar relacionados com a OCD (SLATER et al. 1992; HAZEWINKEL, 1993). Já para NAP e HAZEWINKEL (1994) rações com diferentes níveis protéicos não interferiram no seu aparecimento.

FOX \& WALKER (1993b) relataram que a somatotropina e a tirotropina foram identificadas como fatores etiológicos da OCD. Tais hormônios possuem efeito estimulante no crescimento epifisário e níveis elevados causaram alterações na cartilagem articular de forma similar à observada nessa patologia, como proliferação, hipertrofia e hiperplasia das células cartilaginosas, seguida de degeneração de condrócitos e distúrbios de calcificação e ossificação endocondral. NAP (1993) observou que o nível elevado de somatotropina em cães Dogue Alemão, durante as primeiras 20 semanas de vida, produziram alterações da cartilagem articular, porém tais alterações foram transitórias e não interferiram no aparecimento de OCD. Esse mesmo autor sugeriu o trauma como sendo um fator de distúrbio de crescimento ósseo longitudinal.

Estudos sobre a biomecânica da articulação escápulo-umeral, informaram que a superfície articular da porção caudal da cabeça do úmero estava submetida a maior carga da porção caudal da cavidade glenóide quando em extensão. Forças na articulação, causadas por exercícios vigorosos, levaram a fissuras na cartilagem patologicamente espessada, resultando na formação de retalho de cartilagem na área afetada (SLATER et al. 1992).

Desta forma, o conjunto de vários fatores, como distúrbio da ossificação endocondral e espessamento da cartilagem articular (CORING \& BEALE, 1990); traumatismos (SLATER et al. 1992; ALVARENGA et al. 1993); excesso de massa corpórea, exercícios vigorosos, hereditariedade e suplementação vitamínica-mineral (FOX \& WALKER, 1993b; NAP, 1993) levaram ao desenvolvimento da OCD.

Segundo HOULTON \& COLLINSON (1994) o primeiro sinal clínico foi a claudicação, geralmente unilateral, e diminuição da amplitude dos movimentos do membro afetado, podendo ocorrer atrofia por desuso. Verificaram ainda exacerbação da claudicação após exercícios e ligeira melhora após repouso. A persistência dos sinais clínicos em semanas ou meses, variou com a severidade das lesões. O exame clínico revelou dor à palpação da articulação escápulo-umeral, principalmente quando da hiperextensão e em aproximadamente $50 \%$ dos casos, os animais estavam afetados bilateralmente.

$O$ diagnóstico da OCD baseou-se na associação de fatores como: raça, sexo, idade, alimentação, história clínica e exame físico, mas só foi confirmado após exames radiográficos, onde foram observadas irregularidades, achatamento ou concavidade radiotransparente no contorno articular da porção caudal da cabeça do úmero, normalmente acompanhados de esclerose do osso subcondral (OWENS, 1982).

A progressão da OCD resultou em alterações osteoartríticas representadas por formação de osteófitos nas porções caudais da cavidade glenóide e na cabeça do úmero. Tais lesões foram observadas principalmente nos casos mais avançados ou não tratados (HOULTON \& COLLINSON, 1994).

Há divergências quanto ao melhor tratamento para OCD da cabeça do úmero. Segundo FOX $\&$ WALKER (1993a), apenas $25 \%$ dos animais acometidos tratados conservativamente apresentaram melhora clínica. RUDD et al. (1990) recomendaram que cães com menos de seis meses de idade, sinais clínicos discretos, pequena lesão articular e ausência de fragmento articular livre devem ser tratados conservativamente, com restrição de exercícios sem administração de analgésicos, e redução da ingestão de alimentos e/ou correção da dieta.

De acordo com ALVARENGA et al. (1993), o uso de esteróides e anti-inflamatórios nãoesteroidais reduziram a dor e conseqüentemente a claudicação, mas não promoveram a cura nem retardaram o desenvolvimento das lesões.

VAN RYSSEN et al. (1993) sugeriram a artroscopia como forma de diagnóstico, permitindo ainda intervenção cirúrgica simultânea, oferecendo excelente resultado cosmético e rápido retorno a função, porém necessitando de instrumental específico e grande familiaridade do cirurgião com a técnica.

HOULTON \& COLLINSON (1994) afirmaram que a cirurgia foi o único tratamento eficaz para a $\mathrm{OCD}$, pois eliminou a claudicação e preveniu ou reduziu o desenvolvimento de doença articular degenerativa. A cirurgia consistiu na remoção do retalho ou fragmento articular que irritava a membrana sinovial e superfície articular da cavidade glenóide.

RUDD et al. (1990) recomendaram a curetagem da lesão que foi preenchida posteriormente por fibrocartilagem. FOX \& WALKER (1993a) preconizaram a realização de alguns orifícios no defeito subcondral, utilizando fio de Kirschner, para permitir neovascularização sem perturbar elementos cartilaginosos já presentes. O pós-operatório requer 
restrição de atividades por duas a quatro semanas, com pequenas caminhadas após este período (ALVARENGA et al. 1993). Estudos comparativos entre os tratamentos conservador e cirúrgico demonstraram que o período de recuperação dos casos tratados cirurgicamente foi de aproximadamente um mês, enquanto que os animais que receberam tratamento conservador obtiveram resposta após sete meses (FOX \& WALKER, 1993).

O prognóstico, segundo HOULTON \& COLLINSON (1994), para pacientes tratados cirurgicamente, antes do aparecimento de osteoartrose, é excelente, porém alguns cães podem continuar com discreta claudicação ocasional.

\section{MATERIAIS E MÉTODOS}

Foram estudados 36 casos de OCD da cabeça do úmero em cães, atendidos junto ao serviço de Ortopedia e Traumatologia do Hospital Veterinário da Faculdade de Ciências Agrárias e Veterinárias - UNESP - Campus de Jaboticabal, no período de $1^{\circ}$ de Janeiro de 1991 a 31 de Dezembro de 1996. Os animais, 28 machos e 8 fêmeas, pertenciam a diversas raças, com idades compreendidas entre 5 e 24 meses, pesando entre 15 e 53 quilogramas (Tabela 1).

O principal sinal clínico presente era claudicação de apoio em membro torácico. Durante a anamnese foram obtidos dados quanto ao tipo de alimentação, se caseira ou comercial; suplementação vitamínica-mineral e grau de exercício, sendo considerado exercício forçado aquele realizado com participação do proprietário.

Ao exame clínico os animais exibiram sensibilidade em vários pontos do membro afetado, dificultando as interpretações. O estudo radiográfico simples, em projeção médio-lateral, de ambas as articulações escápulo-umerais, independente da sintomatologia ser uni ou bilateral, revelou alteração do contorno articular na porção caudal da cabeça do úmero afetado. Lesões bilaterais foram encontradas em 17 animais.

Em 9 animais, por vontade do proprietário, optou-se pelo tratamento conservador, sendo indicada restrição a exercícios durante quatro semanas, acompanhada de redução de peso e correção da dieta. Os demais cães foram submetidos à intervenção cirúrgica
Tabela 1 - Distribuição segúndo raça, idade, sexo, peso e localização dos cães portadores de osteocondrite dissecante atendidos junto ao Serviço de Ortope-dia e Traumatologia do Hospital Veterinária da FVAVJ, UNESP, no período de Janeiro de 1991 a Dezembro de 1996.

Número Raça Idade Sexo $\begin{gathered}\text { Massa } \\ \text { Corpórea } \\ (\mathrm{kg})\end{gathered}$

\begin{tabular}{|c|c|c|c|c|c|}
\hline 1 & Fila Brasileiro & 6 meses & M & 32 & D \\
\hline 2 & Fila Brasileiro & 8 meses & M & 35 & D \\
\hline 3 & Rottweiler & 5 meses & M & 24 & $\mathrm{E}$ \\
\hline 4 & SRD & 12 meses & M & 22 & $\mathrm{E}$ \\
\hline 5 & Dogue Alemão & 8 meses & $\mathrm{F}$ & 41 & B \\
\hline 6 & Rottweiler & 10 meses & M & 36 & $\mathrm{E}$ \\
\hline 7 & Rottweiler & 5 meses & $\mathrm{F}$ & 22 & B \\
\hline 8 & Fila Brasileiro & 24 meses & $\mathrm{M}$ & 53 & D \\
\hline 9 & Rottweiler & 6 meses & M & 26 & D \\
\hline 10 & SRD & 7 meses & $\mathrm{F}$ & 28 & D \\
\hline 11 & Rottweiler & 9 meses & M & 37 & B \\
\hline 12 & Mastin Napolitano & 8 meses & M & 50 & B \\
\hline 13 & Rottweiler & 8 meses & M & 37 & B \\
\hline 14 & Pastor Alemão & 10 meses & M & 31 & B \\
\hline 15 & Rottweiler & 7 meses & M & 34 & B \\
\hline 16 & Dogue Alemão & 20 meses & $\mathrm{F}$ & 52 & D \\
\hline 17 & Bernese Mt. Dog & 24 meses & $\mathrm{F}$ & 35 & $\mathrm{D}$ \\
\hline 18 & Rottweiler & 12 meses & $M$ & 50 & B \\
\hline 19 & Rottweiler & 6 meses & M & 35 & $\mathrm{E}$ \\
\hline 20 & Fila Brasileiro & 7 meses & $\mathrm{F}$ & 32 & $\mathrm{E}$ \\
\hline 21 & Fila Brasileiro & 5 meses & $M$ & 27 & B \\
\hline 22 & SRD & 8 meses & M & 15 & B \\
\hline 23 & Mastin Napolitano & 9 meses & M & 42 & $\mathrm{E}$ \\
\hline 24 & Rottweiler & 12 meses & M & 35 & B \\
\hline 25 & Rottweiler & 7 meses & $M$ & 25 & B \\
\hline 26 & Mastin Napolitano & 5 meses & $\mathrm{F}$ & 28 & D \\
\hline 27 & Pastor Alemão & 9 meses & $\mathrm{M}$ & 24 & D \\
\hline 28 & Fila Brasileiro & 12 meses & M & 43 & $\mathrm{E}$ \\
\hline 29 & Pointer Inglès & 8 meses & M & 24 & B \\
\hline 30 & Rottweiler & 6 meses & M & 26 & B \\
\hline 31 & Golden Retriever & 5 meses & $\mathrm{M}$ & 21 & $\mathrm{D}$ \\
\hline 32 & Rottweiler & 8 meses & M & 32 & $\mathrm{E}$ \\
\hline 33 & Rottweiler & 6 meses & M & 26 & B \\
\hline 34 & SRD & 9 meses & $\mathrm{F}$ & 20 & D \\
\hline 35 & Rottweiler & 9 meses & $M$ & 28 & B \\
\hline 36 & Bernese Mt. Dog & 7 meses & M & 23 & B \\
\hline
\end{tabular}

Legenda: M - macho; F - fềmea; D - lado direito; E - lado esquerdo; B bilateral uma ou duas semanas após o diagnóstico, excetuandose um cão, que por deliberação do proprietário, foi operado 240 dias após.

O tratamento cirúrgico, realizado em 27 cães, consistiu na remoção do retalho de cartilagem, regularização das bordas e curetagem da lesão articular, seguida de perfuração do osso subcondral com fio de Kirschner com 1,5 mm em 3 ou 4 pontos no interior do defeito articular. O pós-operatório 
consistiu na administração de antibiótico (amoxicilina $^{\mathrm{a}}$ - 10mg/ $\mathrm{kg} / \mathrm{m} . c . S C$ ) e analgésico (dipirona ${ }^{b}-20 \mathrm{mg} / \mathrm{kg} / \mathrm{m} . c . S C$ ), seguidos de restrição ao exercício por 21 dias. A correção da dieta quando cabível, consistiu na interrupção de qualquer suplemento alimentar e no fornecimento exclusivo de ração comercial em menores quantidades para permitir a redução da massa corpórea dos animais. Os cães com lesões bilaterais foram submetidos a intervenção cirúrgica em ambos os lados, no mesmo dia, sendo realizada uma intervenção subseqüente a outra.

Os dados relativos a evolução dos casos atendidos e tratados foram obtidos, na maioria das vezes por telefone, !c:'ando-se em conta a persistência ou não da claudicação presente antes do tratamento. Os proprietários foram contactados após um período mínimo de uma e máximo de 42 semanas após a terapia.

\section{RESULTADOS E DISCUSSÃO}

A Tabela 1 demonstra a distribuição dos animais segundo a raça, idade, sexo, massa corpórea e localização da lesão; observando-se que a doença acometeu cães de raças grandes, assim como citaram OLSSON (1993) e HOULTON \& COLLINSON (1994); exceção a um cão sem raça definida (caso $n^{\circ}$ 22).

O peso dos cães acometidos variou entre 15 e $53 \mathrm{~kg}$, com $75 \%$ dos cães $(n=27)$ pesando entre 25 e $50 \mathrm{~kg}$, assim como mencionado por RUDD et al. (1990). A idade de ocorrência das manifestações clínicas deu-se entre 5 e 24 meses, sendo que $61,1 \%$ dos cães acometidos $(n=22)$ tinham idade entre 4 e 8 meses, a semelhança do observado por HOULTON \& COLLINSON (1994).

Observou-se maior freqüência desta artropatia em cães machos, na proporção de 3,5 machos para uma fêmea, concordando com o relatado por SLATER et al. (1992). Tal fato deve-se principalmente pelo crescimento precoce e acelerado de cães machos, atingindo maior massa corpórea em menor espaço de tempo.

$\mathrm{O}$ acometimento bilateral em $47.2 \%$ dos cães $(n=17)$, confirma observações de HOULTON \& COLLINSON (1994) que descreveram ocorrência em cerca de $50 \%$. Em nossa opinião, o acometimento bilateral pode ocorrer devido ao fato da calcificação endocondral e o conseqüente crescimento epifisário acontecerem simultaneamente nos dois membros, que associados à presença de fatores genéticos $\mathrm{e}$ ambientais, desencadeiam complicações articulares bilaterais.
A raça Rottweiler apresentou maior incidência de OCD $(n=15)$, quando comparada as demais envolvidas neste estudo; como também relataram HOULTON \& COLLINSON (1994). A provável explicação para tal representação racial deve-se ao grande número de cães desta raça, que vem sendo difundida entre os proprietários de animais de estimação nos últimos anos, sendo esta uma tendência mundial. Observou-se também elevada incidência na raça Fila Brasileiro $(n=6)$, ainda pouco citada por outros autores; seguida por cães sem raça definida $(n=4)$, Mastim Napolitano $(n=3)$, Dogue Alemão $(n=2)$, Pastor Alemão $(n=2)$, Bernese Mountain Dog $(n=2)$, Golden Retriever $(n=1)$ e Pointer Inglês $(n=1)$.

É importante ressaltar a ocorrência, na casuística aqui relatada, de OCD bilateral em um cão sem raça definida de $15 \mathrm{~kg}$ de massa corpórea, oriundo de canil público, onde seguramente não desfrutou de hipernutrição ou suplementação. $\mathrm{O}$ acometimento bilateral neste caso pode estar relacionado a fatores traumáticos, porém se torna dificil afirmar tal hipótese, uma vez que a história anterior a adoção por parte de um proprietário, não era conhecida.

De acordo com a literatura, verifica-se que a etiologia da OCD tem caráter multifatorial. Alguns autores relacionaram o surgimento das alterações articulares com seleção racial (OLSSON, 1993), massa corpórea (RUDD et al. 1990), sexo (HOULTON \& COLLINSON, 1994), componentes da dieta (SLATER et al. 1992), altas concentrações séricas de somatotropina e tirotropina (FOX \& WALKER, 1993b; NAP, 1993), efeitos biomecânicos articulares (SLATER $\boldsymbol{e}$ al. 1992) e traumatismos (ALVARENGA et al. 1993).

No presente trabalho observou-se que cinco cães $(13,9 \%)$ recebiam alimentação comercial, dois alimentação caseira $(5,5 \%)$ e $29(80,6 \%)$ recebiam alimentação comercial adicionada de suplementação alimentar (principalmente vitaminas A e D, e farináceos). A maioria, portanto foi hipernutrida, e, segundo os proprietários, a partir do desmame, ao redor dos 60 dias de idade, período em que os animais tornaram-se muito obesos. O principal fator desencadeante desta artropatia em nossa opinião foi esse desequilíbrio nutricional, imposto durante a fase inicial de crescimento, associado a fatores ambientais ou genéticos, concordando com as observações de SLATER et al. (1992).

No tocante aos achados radiográficos, observou-se similitude aos descritos por OWENS (1982). As lesões mais freqüentes eram representadas por concavidade radiotransparente na porção caudal da cabeça do úmero (Figura 1) e em nenhuma radiografia foi evidenciado fragmento articular livre. 


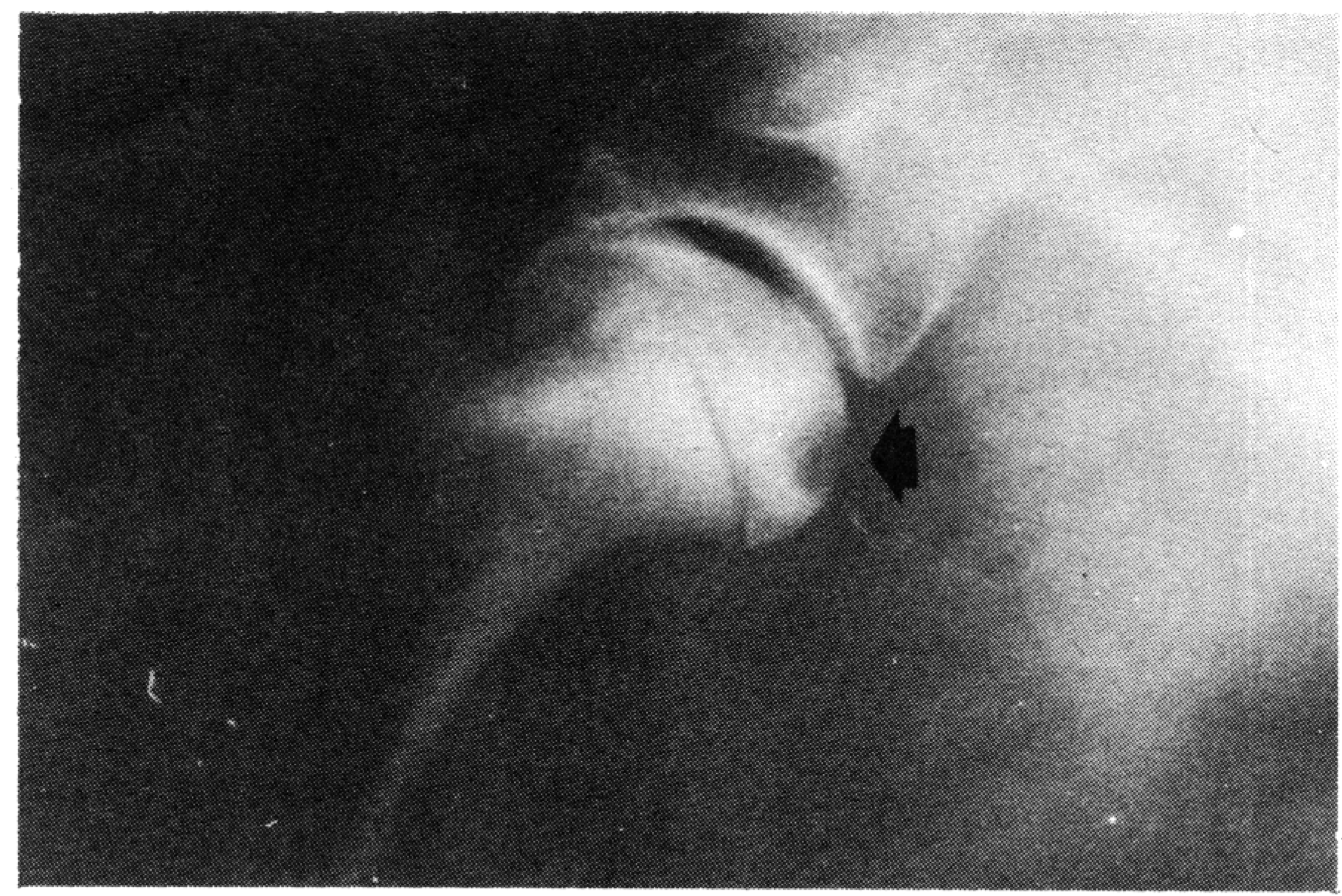

Figura 1 - Imagem radiográfica de osteocondrite dissecante em cão Fila Brasileiro, macho, com 8 meses de idade (caso n. 2). Observa-se lesão radiotransparente de forma discóide junto ao osso subcondral da porção caudal da superfície articular da cabeça do úmero direito

Entre os animais tratados conservativamente, $88,9 \%(n=8)$ continuaram claudicando com freqüência moderada, segundo o proprietário, e apenas $11,1 \%(\mathrm{n}=1)$ deles apresentaram ausência do sintoma inicial, discordando das informações de FOX \& WALKER (1993a), que observaram melhora em $25 \%$ dos casos. Na presente casuística, supõe-se que os proprietários não impuseram repouso e dieta adequada aos seus animais.

Em todos dos animais tratados cirurgicamente $(n=25)$ houve completa remissão dos sinais clínicos entre uma e sete semanas de pós-operatório. Resultados semelhantes foram citados também por HOULTON \& COLLINSON (1994).

\section{CONCLUSÕES}

Através da análise comparativa dos resultados obtidos no presente trabalho, concluiu-se que:

os cães afetados pela osteocondrite dissecante são representantes, em sua maioria, de raças de grande porte, crescimento rápido e a maior porcentagem dos animais acometidos recebiam suplementação vitaminica-mineral;

o tratamento cirúrgico é o procedimento de eleição a ser tomado face a esta artropatia;

por ocasião do esquema inicial de vacinação, os clínicos Veterinários conhecedores do assunto devem orientar os proprietários de cães de raças grandes, sobre os fatores nutricionais envolvidos na gênese da osteocondrite dissecante.

\section{FONTES DE AQUISIÇÃO}

a - Clamoxyl LA. Smithkline \& Beecham.

b - Novalgina. Hoechst do Brasil.

\section{REFERÊNCIAS BIBLIOGRÁFICAS}

ALVARENGA, J., GARCIA, J.A., IWASAKI, M., et $a$ Osteocondrite dissecante da cabeça do úmero em cães. A Hor Veterinária, v. 73, p. 68-74, 1993.

CORING, R.L., BEALE, B.S. Failure arthroscopic dislodgement of cartilage flaps for treatment of osteochondrosis in the shoulder of two racing greyhounds. Journal American Animal Hospital Association, v. 26, p. 413-416, 1990.

FOX, S.M., WALKER, A.M. OCD of the humeral head: its diagnosis and treatment. Veterinary Medicine, v. 2, p. 123131, 1993a.

FOX, S.M., WALKER, A.M. The ethiopathogenesis of osteochondrosis. Veterinary Medicine, v. 2, p. 116-122, $1993 b$

HAZEWINKEL, H.A.W. Nutrition in orthopedics. In: BOJRAB, M.J. Disease mechanisms in small animal surgery. 2 ed Philadelphia. Lea \& Febiger, 1993. Cap. 149, p. 1119-1128.

NAP, R.C. Nutritional influences on growth and skeletal development in the dog. Utrecht - Holland. 98 p. Tese (PhD)Utrecht University, 1993.

NAP, R.C., HAZEWINKEL, H.A.W. Growth and skeletal development in the dog in relation to nutrition: A review. Veterinary Quarterly. v. 16, p. 50-59, 1994.

OLSSON, S.E. Pathophysiology, morphology and clinical signs of osteochondrosis in the dog. In: BOJRAB, M.J. Disease mechanisms in small animal surgery. 2 ed. Philadelphia, Lea \& Febiger, 1993. Cap. 111, p. 777-798.

OWENS, J.M. Radiographic interpretation for the small animal clinician. Saint Louis: Ralston Purina, 1982. Cap. 3: The joints: p. 29-32.

HOULTON, J.E.F., COLLINSON, R.W. Manual of small animal arthrology. 1 ed. London. British Small Animal Veterinary Association. Cap. 6: Osteochondrosis: p. 75-85. 1994.

RUDD, G.G., WHITEHAIR, J.G., MARGOLIS, J.H. Results of management of osteochondritis dissecans of the humeral head in dogs: 44 cases (1982-1987). Journal American Animal Hospital Association, v. 26, p. 173-178, 1990.

SLATER, M.R., SCARLET, J.M., DONOGHUE, S., et al. Diet and exercise as potential risk factors for osteochondritis dissecans in dogs. American Journal Veterinary Research, v. 53, n. 11, p. 2119-2124, 1992.

VAN RYSSEN, B., VAN BREE, H., VYT, P. Arthroscopy of the shoulder joint in the dog. Journal American Animal Hospital Association, v. 29, p. 101-105, 1993. 